\title{
Original
}

\section{The P2 Promoter of the CREM Gene is Responsive to cAMP-PKA Signaling Pathway in Osteoblastic MC3T3-E1 Cells}

\author{
Yu-Feng Huang ${ }^{1,2)}$ and Barbara E. Kream ${ }^{3)}$
}

${ }^{1)}$ College of Oral Medicine, Chung Shan Medical University, Taichung, Taiwan

${ }^{2}$ Center of Oral Medicine, Chung Shan Medical University Hospital, Taichung, Taiwan

${ }^{3}$ Department of Medicine, School of Medicine, University of Connecticut Health Center, Connecticut, USA

(Accepted for publication, September 21, 2011)

\begin{abstract}
The expression of inducible cAMP early repressor (ICER) has been demonstrated in cultured osteoblastic MC3T3-E1 cells, calvarial cultures and in vivo, mainly via the cAMP-PKA signaling pathway. To determine the molecular mechanism(s) for this action, a fragment of the CREM P2 promoter from -238 bp to +14 bp was linked to a luciferase reporter (CREMP2-Luc238). This region contains 2 clusters of cAMPresponsive element (CRE). The 5' cluster contains CRE1 and CRE2 while the 3' cluster contains CRE3 and CRE4 and the two clusters are separated by $12 \mathrm{bp}$. In order to study if any or all CREs are responsive to cAMP induction, osteoblastic MC3T3-E1 cells were stably transfected with constructs having serial deletions of each CRE or mutations in each cluster of the CREMP2-Luc construct. Agonists that stimulate the cAMP-PKA pathway induced CREMP2-Luc238 activity, and neither PKC nor $\mathrm{Ca}^{++}$pathways did. The deletion of CRE1 and CRE2 did not significantly abolish the cAMP inducibility of CREMP2-Luc238 activity. Electrophoretic mobility shift assay showed many DNA/protein complexes when probes from either CRE1-2 or CRE3-4 were incubated with FSK-treated nuclear extract from MC3T3-E1 cells. However, only a small portion of the complexes was competed by a consensus CRE oligonucleotide. Interestingly, a major DNA/protein complex seen in the binding of CRE3-4 was not competed by the consensus CRE oligonucleotide, but by a mutated CRE oligonucleotide suggesting that other transcription factor(s) may be involved in the binding of CRE3-4. The CRE binding DNA/protein complexes were supershifted mainly by antibodies against ICER and CREB, mildly by C/EBPß suggesting these transcription factors may be involved in the FSK induction of the P2 promoter of the CREM gene.
\end{abstract}

Key words: CREM, CRE, Transfection, EMSA, cAMP

\section{Introduction}

The CREM gene, cAMP responsive element modulator, encodes multiple isoforms of transcription factors depending on the promoters driving the expression. The inhibitory isoforms, including $\alpha, \beta$ and $\gamma$ contain phosphorylation P-box region, DNA and dimerization domains. On the other hand, the activators, the ô isoforms, contain additional glutamine-rich transactivation domain. All these isoforms of the CREM gene are transcribed from an upstream GC-rich promoter (P1) and constitutively expressed in a tissue specific and developmentally-regulated pattern $^{1-3)}$. The hallmark of CREM biology is the switchover from inhibitory to stimulatory isoforms in the testis upon the onset of spermatogenesis, a process that is due to a change in tissue specific alternative splicing and directed by follicle stimulating hormone. It is not surprising, therefore, that CREM male knockout mice

Correspondence to: Yu-Feng Huang DDS, MSD, PhD.,College of Oral Medicine Chung Shan Medical University No.110, Section 1, Jian-Kuo North Road Taichung City, TAIWAN, E- mai I : whuang@smu. edu. tw completely lack mature sperm and are sterile.

Another isoforms have been isolated recently, $\theta 1$ and $\theta 2$, which are inducible by cAMP signaling pathway and are transcribed from an intronic promoters, the $\mathrm{P} 3$ or $\mathrm{P} 4$ promoters, respectively 3). The functions of CREM $\theta 1$ and $\theta 2$ have been demonstrated to be stimulatory. On the other hand, the inducible cAMP early repressor (ICER), encodes a $15 \mathrm{kDa}$ protein and is a cAMP inducible member of the CREM transcription factor superfamily 2). It is transcribed from another alternative intronic promoter, the P2 promoter. Therefore, ICER transcripts only contain basic region for DNA binding and leucine-zipper dimerization domain. ICER was originally discovered in pituitary gland and was studied on different in vitro system. Most of these studies showed that ICER delivers different functions in different system. For example, in neuroendocrine tumor cells, ICER is anti-proliferative ${ }^{4)}$, while it is anti-apoptotic in T cells ${ }^{5,6)}$. In our laboratory, we have reported that ICER mRNA and protein are induced by PTH in cultured osteoblastic cells and mouse calvarial cultures and in 


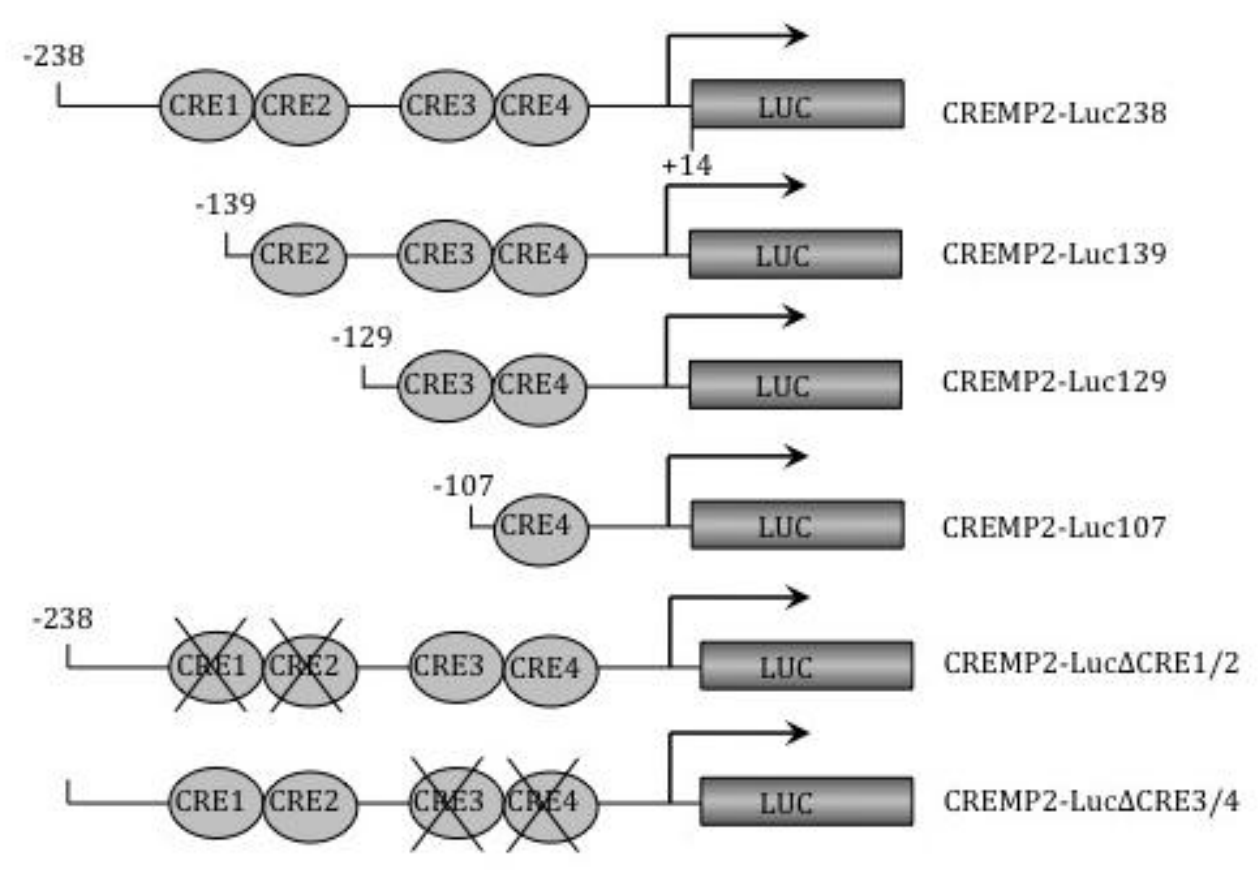

Figure 1 Schematic diagram of the CREM P2 promoter-luciferase construct

vivo mainly via a cAMP-PKA signaling pathway ${ }^{7,8)}$. Since PTH shows both anabolic and catabolic effects on bone depending on the mode of administration, which requires regulation of gene expression mainly through cAMP-PKA signaling pathway, it becomes very important to study the regulation mechanism of ICER by PTH to understand the mechanisms involved in PTH functions.

The first aim of this study was to demonstrate the signaling pathway involved in the transcriptional regulation of ICER by PTH. The second aim was to characterize the responsive elements and transcription factors involved in the cAMP-dependent transcription of the CREM P2 promoter.

\section{Materials and Methods}

\section{Reagents}

PTH(1-34) was purchased from Sigma Chemical Inc, and was diluted in a vehicle containing bovine serum albumin in a slightly acidic condition. Forskolin (FSK), phorbol myristatic acid (PMA) and ionomycin(iono) were obtained from Biomol Co, and were constituted with absolute ethanol. 8-bromo-cAMP (8-Br-cAMP) was purchased from Sigma Chemicals Inc, and was constituted with culture medium. H89 was from Biomol and was diluted in absolute ethanol.

\section{Constracts}

A fragment of the CREM P2 promoter was kindly provided by Dr. C. Molina (UNJMD, Newark, NJ) which is from -238 bp to +14 bp. The promoter fragment was cloned into a pGL3 vector containing a luciferase reporter (CREMP2-Luc238). Within the promoter fragment, there are $4 \mathrm{CRE}$-like binding sites positioned in 2 clusters with each CRE within the cluster spaced by 3 bp (Figure 1).

To generate promoter constructs with 5' serial deletion, sitespecific primers were synthesized and the deletion constructs were generated by PCR. The PCR products were eventually cloned to the pGL3 vector containing the luciferase reporter. CREMP2Luc139 lacks the most 5' CRE (CRE1), while CREMP2-Luc129 had additional removal of CRE2. CRE1 to CRE3 were removed in CREMP2-Luc107 leaving only an intact CRE4. Constructs with serial deletion were all sequence confirmed (Figure 1).

Mutagenesis of each CRE cluster was performed with a sitespecific mutagenesis kit (Invitrogen) following the manufactural instruction. In brief, oligonucleotides containing specific mutations were synthesized and PCR was performed using CREMP2-Luc238 circular plasmid as a template. The PCR products were digested with Dpn I and the constructs were purified with Qiagen plasmid preparation (Qiagen, Inc., Valencia, CA, USA). CREMP2-Luc $\Delta$ CRE1-2 and CREMP2-Luc $\Delta$ CRE3-4 were obtained representing mutations at CRE1-2 and CRE3-4, respectively (Figure 1). Both constructs were sequenced to verify the containing of desired mutations.

\section{Stable transfection}

All constructs were stably transfected into osteoblastic MC3T3E1 cells ${ }^{9)}$. In brief, MC3T3-E1 cells were plated at 200,000 cells/ well in a $35-\mathrm{mm}$ plate overnight. Stable transfection was carried 
Yu-Feng Huang et al.: The P2 Promoter of the CREMGene is Responsive to cAMP-PKA Signaling Pathway

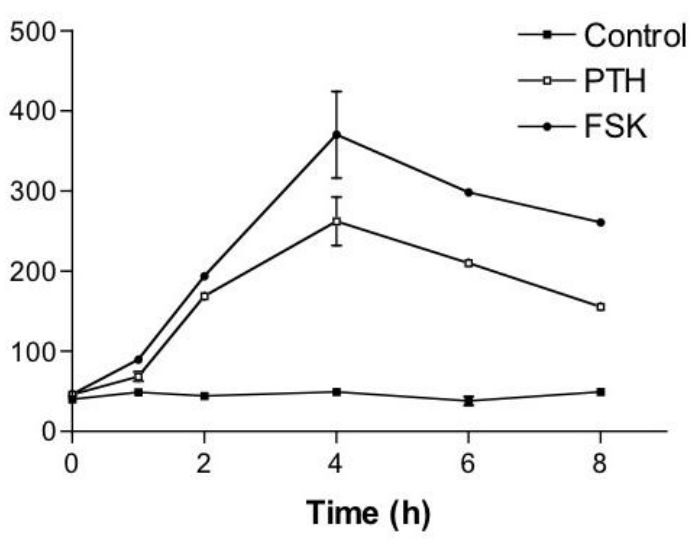

Figure 2 Time course of PTH and FSK treatment

out by using lipoFECTAMINE reagent (Invitrogen). After 6 weeks selection with G418, two separate populations were obtained with each population containing multiple clones of transfectants.

\section{Luciferase assay}

Stably transfected cells were plated at 5000 cells/well in a 35mm plate in DMEM supplemented with $10 \%$ heat inactivated fetal bovine serum, $1000 \mathrm{U} / \mathrm{ml}$ penicillin, $500 \mathrm{U} / \mathrm{ml}$ streptomycin, and 1:1000 G418. Confluent cells were treated with 10nM PTH(134) or signaling molecules. The induced CREMP2 expression was measured as luciferase activity normalized to total protein content estimated by the indicator bicinchoninic acid ${ }^{10)}$. The assay was performed as triplicate samples for each treatment group and all constructs were studied at least 4 times.

\section{Nuclear extract preparation}

Nuclear extracts were prepared as previously described ${ }^{11)}$ with some modifications. Briefly, MC3T3-E1 cells were treated with FSK for up to $2 \mathrm{~h}$, washed with cold PBS, scraped and resuspended in $2 \mathrm{ml}$ cold PBS. Cells were pelleted by centrifugation at $500 \mathrm{X}$ $\mathrm{g}$ at $4^{\circ} \mathrm{C}$ and resuspended in buffer A (10 mM HEPES-KOH, $\mathrm{pH}$ 7.9, $0.75 \mathrm{mM}$ spermidine, $0.15 \mathrm{mM}$ spermine, $0.1 \mathrm{mM}$ EDTA, 10 $\mathrm{mM} \mathrm{KCl}$ and freshly added $0.5 \mathrm{mM}$ dithiothreitol, $0.5 \mathrm{mM}$ phenylmethylsulfonyl fluoride, $1 \mu \mathrm{g} / \mathrm{ml}$ leupeptin and $1 \mu \mathrm{g} / \mathrm{ml}$ pepstatin). Cells were re-pelleted, resuspended in buffer A and homogenized. Homogenates were centrifuged for $10 \mathrm{~min}$ at 16000 $\mathrm{X}$ g at $4^{\circ} \mathrm{C}$ and pellets were resuspended in $0.5 \mathrm{ml}$ of buffer $\mathrm{B}$ (20 mM HEPES-KOH, pH 7.9, 20\% v/v glycerol, $0.42 \mathrm{mM} \mathrm{NaCl,}$ $0.75 \mathrm{mM}$ spermidine, $0.15 \mathrm{mM}$ spermine, $0.2 \mathrm{mM}$ EDTA and freshly added $0.5 \mathrm{mM}$ dithiothreitol, $0.5 \mathrm{mM}$ phenylmethylsulfonyl fluoride, $1 \mu \mathrm{g} / \mathrm{ml}$ leupeptin and $1 \mu \mathrm{g} / \mathrm{ml}$ pepstatin) and homogenized. Homogenates were left at $4^{\circ} \mathrm{C}$ for $30 \mathrm{~min}$ and centrifuged for $30 \mathrm{~min}$ at $25,000 \mathrm{X}$ g at $4^{\circ} \mathrm{C}$. Supernatants were collected, dialyzed for $6 \mathrm{~h}$ against $250 \mathrm{ml}$ of buffer C containing $20 \mathrm{mM}$ HEPES-KOH pH 7.9, 20\% glycerol (v/v), $0.1 \mathrm{mM} \mathrm{NaCl}, 0.2 \mathrm{mM}$ EDTA, $0.5 \mathrm{mM}$ dithiothreitol, 0.5
mM phenylmethylsulfonyl fluoride, $1 \mu \mathrm{g} / \mathrm{ml}$ leupeptin and $1 \mu \mathrm{g} /$ $\mathrm{ml}$ pepstatin. Nuclear proteins were quantified by BCA assay ${ }^{10}$ ) and stored at $-70^{\circ} \mathrm{C}$.

\section{Electrophoretic mobility shift assay}

Ten picomole of each complementary deoxyoligonucleotides were separately incubated with $5 \mathrm{X}$ kinase forward reaction buffer, [g- ${ }^{32} \mathrm{P}$ ]ATP (3000 Ci/mmol, New England Nuclear Research Products, DuPont Co, Wilmington, DC) and $\mathrm{T}_{4}$ DNA kinase (GIBCO, Grand Island, NY) for $1 \mathrm{~h}$ at $37^{\circ} \mathrm{C}$. The two reactions were mixed, boiled, and left at room temperature to allow proper annealing. The resulting double-stranded oligonucleotide probes were extracted with phenol/chloroform, and precipitated with ethanol in the presence of $2.5 \mathrm{M}$ ammonium acetate. After pelleting, the probes were dissolved in $50 \mathrm{ml} \mathrm{H}_{2} \mathrm{O}$. Nuclear extract or purified CREB-bZip DNA binding domain (Santa Cruz Biotechnology, Santa Cruz, CA) was used in a 20-ml binding reaction in the presence of $20 \mathrm{mM}$ HEPES pH 7.9, $50 \mathrm{mM} \mathrm{KCl}$, $6.25 \mathrm{mM} \mathrm{MgCl}$, $0.5 \mathrm{mM}$ EDTA, $10 \%$ glycerol $^{12)}$, 1mM DTT and $0.1 \mathrm{mg} / \mathrm{ml}$ poly(dI-dC)·poly(dI-dC) (Pharmacia Biotech, Piscataway, $\mathrm{NJ}$ ), and $10^{5} \mathrm{cpm}$ deoxyoligonucleotide probes at room temperature for $30 \mathrm{~min}$. In the competition experiments, the CRE and C/EBP consensus oligonucleotides as well as a mutated CRE oligonucleotide (Santa Cruz Biotechnology, Santa Cruz, CA) were incubated with nuclear extract for 15 min followed by the addition of radiolabeled probes for $15 \mathrm{~min}$. In the supershift experiments, probe and nuclear extract were incubated at room temperature for 30 min followed by the addition of specific antibodies against various transcription factors. All antibodies were obtained from Santa Cruz Biotechnology, Santa Cruz, CA. The reactions were fractionated on a $5 \%$ polyacrylamide gel electrophoresis in $0.5 \mathrm{X}$ TBE buffer for $90 \mathrm{~min}$ at $150 \mathrm{~V}$. Gels were placed on a piece of dry $3 \mathrm{M}$ blotting paper, fixed in 10\% acetic acid/10\% methanol for $30 \mathrm{~min}$, dried at $60^{\circ} \mathrm{C}$ for $75 \mathrm{~min}$, and exposed to radiographic films at room temperature.

\section{Results}

\section{CREMP2-Luc238 was induced by PTH mainly through cAMP- PKA signaling pathway}

To determine if PTH induces CREMP2-Luc238 expression in a time-dependent manner, MC3T3-E1 cells stably transfected with CREMP2-Luc238 were grown to confluence and treated with PTH $(10 \mathrm{nM})$ or FSK $(10 \mu \mathrm{M})$ for up to $8 \mathrm{~h}$ in the presence of heatinactivated fetal bovine serum. CREMP2-Luc238 expression was measured as luciferase activity, which was normalized to total protein content. CREMP2-Luc238 activity was highly induced by PTH or FSK in a time-dependent manner, which peaked at 4 h. Thus, CREMP2-Luc238 was treated with signaling effectors in all experiments for $4 \mathrm{~h}$ (Figure 2).

To determine the signaling pathways involved in the induction of CREMP2-Luc238 expression, cells stably transfected with 
Signaling

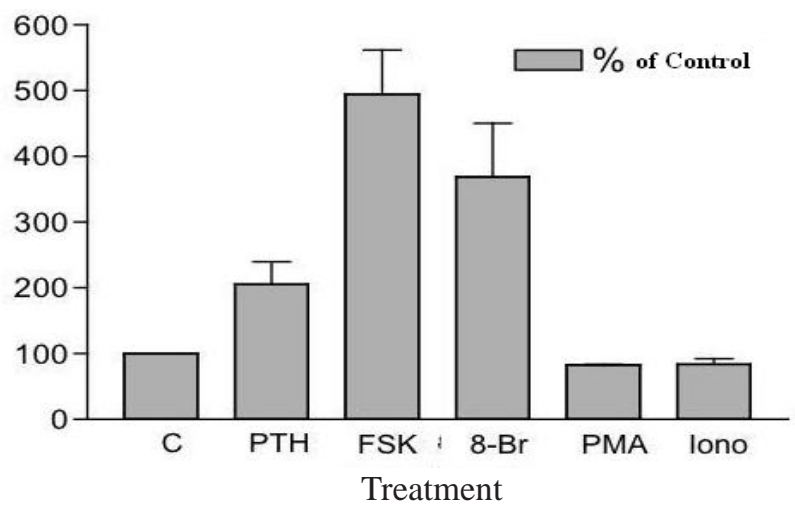

Figure 3A The activity of the CREMP2-Luc238 by various signaling molecules at 4-h treatment

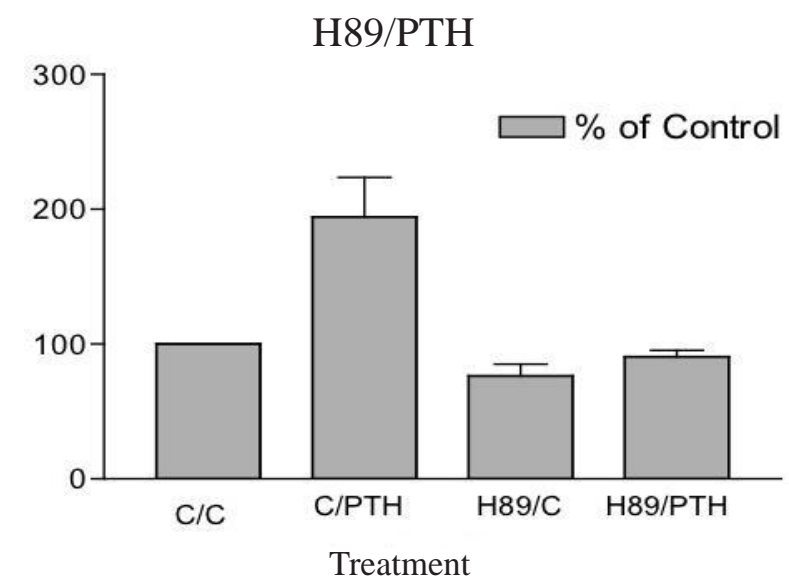

Figure 4 The cAMP inducibility of CREMP2-Luc238 by H89 pretreatment

CREMP2-Luc238 were treated with various pharmacological effectors for $4 \mathrm{~h}$ and the promoter activity was measured as luciferase activity. CREMP2-Luc238 expression was significantly induced by 10nM PTH, $10 \mu \mathrm{M}$ FSK, and 3mM 8-bromo-cAMP (8-Br) to $205 \pm 35 \%, 494 \pm 68 \%$ and $369 \pm 81 \%$ of control, respectively, whereas $100 \mathrm{nM}$ PMA, $1 \mu \mathrm{M}$ ionomycin (Iono) (Figure 3A) and 100nM PTH(3-34) did not (Figure 3B).

To further demonstrate that CREMP2-Luc238 activity was induced by PTH mainly via the cAMP-PKA signaling pathway, cells stably transfected with CREMP2-Luc238 were pre-treated with $30 \mu \mathrm{M}$ H89, a specific PKA inhibitor, and $1 \mathrm{~h}$ before subsequent treatment with PTH for $4 \mathrm{~h}$. The results showed that PTH inducibility of the CREMP2-Luc238 was significantly abolished by H89 pretreatment (Figure 4, H89/PTH).

The data described above suggested that the PTH inducibility of the CREMP2-Luc238 was mediated mainly via the cAMPPKA signaling pathway. In order to determine the involvement of each CRE-like responsive element in the PTH induction, deletions and mutations of the CREM P2 promoter fragment were

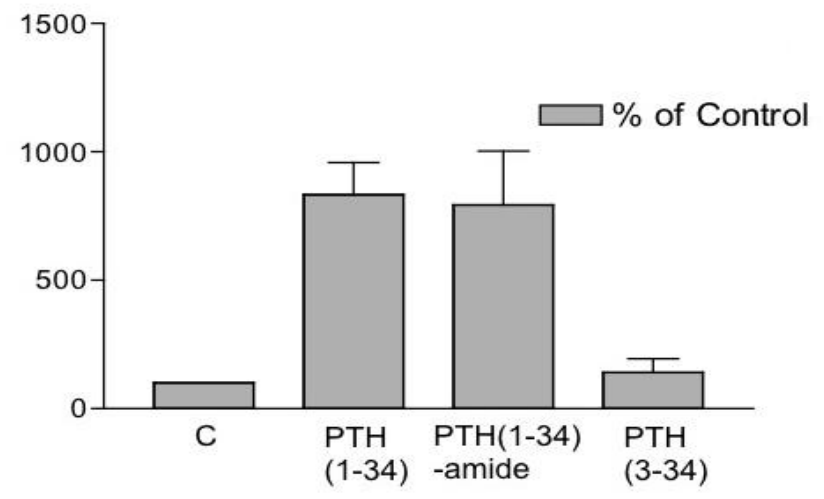

Figure 3B The activity of CREMP2-Luc238 by either PTH(1-34) or $\operatorname{PTH}(3-34)$

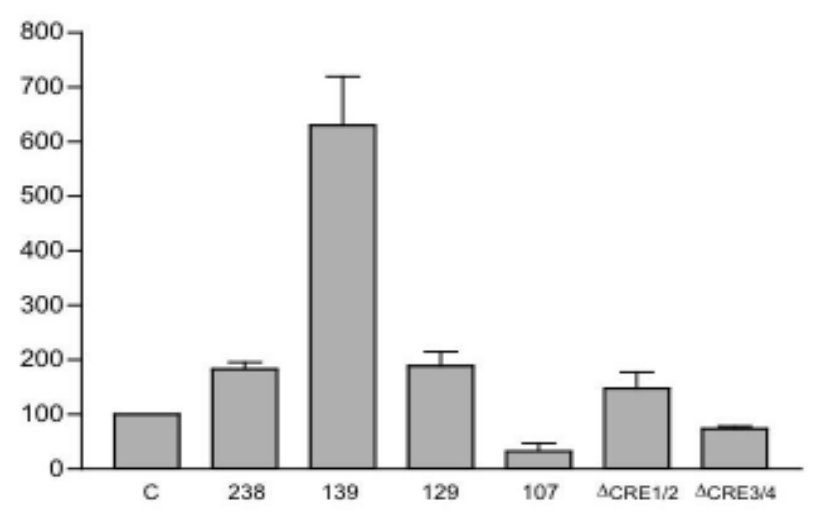

Figure 5 The cAMP-inducibility of various CREMP2-Luc constructs

generated as described in the Materials and Methods. All constructs were stably transfected into MC3T3-E1 cells. After induction with $10 \mu \mathrm{M}$ FSK, deletion of the CRE1 (CREMP2Luc139) did not reduce but significantly enhanced cAMP induction of the promoter activity (Figure 5). Further deletion of the CRE2 (CREMP2-Luc129) did not show any alteration in the inducibility of the FSK compared to CREMP2-Luc238 (Figure 5). However, additional removal of the CRE3 (CREMP2-Luc107) completely reduced the FSK inducibility (Figure 5). Mutations at CRE1/2 (CREMP2-Luc238 $\Delta$ CRE1/2) did not alter significantly the FSK inducibility of the promoter activity. However, mutations at CRE3/4 (CREMP2-Luc238 $\triangle$ CRE3/4) significantly reduced FSK induction of the promoter activity (Figure 5). The data suggested that CRE3/4 plays a more responsive role in cAMP induction of the CREMP2-Luc238 activity.

The EMSA of the nuclear extracts from FSM-induced MC3T3E1 cell formed at least $4 \mathrm{DNA}$ /protein complexes with radiolabeled CRE1/2 probe (Figure 6). Complex I was competed by a 
Yu-Feng Huang et al.: The P2 Promoter of the CREMGene is Responsive to cAMP-PKA Signaling Pathway

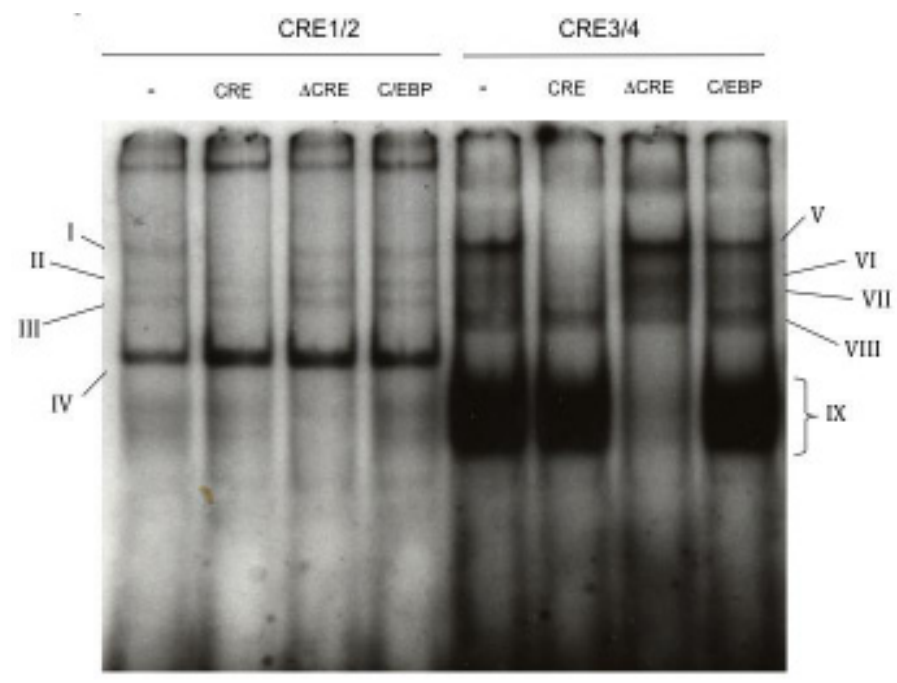

Figure 6 EMSA with various oligo fragments

consensus CRE oligonucleotide (Santa Cruz Biotechnology, Inc., Santa Cruz, CA) and not by either a mutated CRE or C/EBP oligonucleotides (Santa Cruz Biotechnology, Inc., Santa Cruz, CA). None of the remaining DNA/protein complexes (complexes II, III and IV) were competed by these and other consensus oligonucleotides, such as AP-1 and NF- $\mathrm{KB}$ (data not shown). Similarly, the FSK-induced nuclear extract of MC3T3-E1 cells formed at least 5 different DNA/protein complexes with radiolabeled CRE3/4 and only complex $\mathrm{V}$ was competed by the consensus CRE oligonucleotide. Although the DNA/protein binding patterns were similar using CRE1/2 and CRE3/4 probes, CRE3/4 formed additional complexes with the nuclear extracts as shown in Figure 6, complexes IIIV and IX. The intensity of complex IX suggested that there was a significant protein binding with the CRE3/4 and it was competed by the mutated CRE oligonucleotide (Figure 6).

\section{Discussion}

This minimal fragment of the CREM P2 promoter contains only 2 clusters of CREs. The results of this study were in agreement with others ${ }^{2,7)}$ that this promoter does not respond to other stimuli, including PKC and $\mathrm{Ca}^{++}$ionophore, but only to cAMP-PKA signaling pathway. Therefore, this promoter fragment could be a good model to study cAMP-PKA pathway.

The responsiveness of the CREs in the P2 promoter of the CREM gene has been briefly studied ${ }^{2}$. In that study, CRE1-2 and CRE3-4 are separately cloned upstream of the thymidine kinase minimal promoter in pBLCAT2 linked to a CAT reporter and show strong inducibility by CAMP, with CRE3-4 showing more responsiveness than CRE1-2. Our results were in agreement with their report that the CREM P2 promoter fragment was exclusively responsive to cAMP-PKA signaling pathway, in addition that CRE1-2 and CRE3-4 are both responsive to cAMP induction. Our study further characterized the involvement of each CRE in cAMP inducibility by serial deletion of the P2 promoter fragment and mutations of either CRE cluster. The data showed that CRE3 was the most significant responsive element in mediating cAMP induction of the CREMP2-Luc activity since deletion of CRE1-2 (CREMP2-Luc129) did not loose any cAMP inducibility while additional removal of CRE3 (CREMP2-Luc107) significantly abolished the promoter activity, both baseline as well as cAMP inducibility. Interesting to note was that when only CRE1 was deleted, cAMP inducibility was significantly increased suggesting that one or more transcription suppressors may be involved in the binding of CRE1 or the region between -238 bp and -139 bp. Brief computer search of this region did not reveal any consensus DNA sequences for known transcription factors. EMSA using different probes, CRE1-2 and CRE3-4, along with FSK-treated nuclear extract demonstrated different DNA/protein binding patterns suggesting that there may be different groups of transcription factors involved in the binding of CRE1-2 and CRE34. CRE3-4 probe always generated a more intense binding pattern which may reflect that CRE3-4 plays a more direct effect on the response of the CREM P2 promoter activity. Many DNA/protein complexes were observed and only 2 of them were competed by a consensus CRE oligonucleotide. Interestingly, a major DNA/ protein complex (complex IX in Figure 6) was observed mainly with the CRE3-4 probe, and was competed by a commercially available mutated CRE oligonucleotide (Santa Cruz Biotechnology, Inc., Santa Cruz, CA, USA). The EMSA analysis was repeated at least 4 times using different preparations of nuclear extracts from FSK-treated MC3T3-E1 cells with similar results. Preliminary searching the transcription factors involved in the binding of the mutant CRE oligonucleotide did not yield any 
J.Hard Tissue Biology Vol. 20(3):225-230, 2011

results. This suggested that an unidentified transcription factor is involved in the binding of CRE3-4 which, based on the intensity of the complex, may play a major role in the expression of the CREMP2-Luc activity. Further study to characterize the protein is necessary.

\section{References}

1. Lalli E, Lee JS, Lamas M, Tamai K, Zazopoulos E, Nantel F, Penna L, Foulkes NS and Sassone-Corsi P. The nuclear response to cAMP: role of transcription factor CREM. Philos Trans R Soc Lond B Biol Sci 351: 201-209, 1996

2. Molina CA,Foulkes, NS, Lalli E, and Sassone-Corsi P. Inducibility and negative autoregulation of CREM: An alternative promoter directs the expression of ICER, an early response repressor. Cell 5: 875-886,1993

3. Daniel PB, L Rohrbach and JF Habener. Novel cyclic adenosine 3',5'-monophosphate (cAMP) response element modulator theta isoforms expressed by two newly identified cAMP-responsive promoters active in the testis. Endocrinology 141: 3923-3930,2000

4. Razavi R, Ramos JC, Yehia G, Schlotter F and Molina CA. ICER-IIgamma is a tumor suppressor that mediates the antiproliferative activity of cAMP. Oncogene 17: 30153019,1998

5. Ruchaud S, Seite P, Foulkes NS, Sassone-Corsi P and Lanotte M.The transcriptional repressor ICER and cAMP-induced programmed cell death. Oncogene 15: 827-836,1997

6. Bodor J, Bodorova J, Bare C, Hodge DL, Young HA and
Gress RE. Differential inducibility of the transcriptional repressor ICER and its role in modulation of Fas ligand expression in T and NK lymphocytes. Eur J Immunol 32: 203-212, 2002

7. Tetradis S, Nervina JM, Nemoto K and Kream, BE. Parathyroid hormone induces expression of the inducible cAMP early repressor in osteoblastic MC3T3-E1 cells and mouse calvariae. J Bone Miner Res 13: 1846-1851, 1998

8. Nervina JM, Tetradis S, Huang Y-F, Harrison,D, Molina C, and Kream BE. Expression of inducible cAMP early repressor is coupled to the cAMP-protein kinase A signaling pathway in osteoblasts Bone 32(5): 483-490, 2003

9. Huang Y, J Harrison and B Kream. The Role of Proximal cAMP Responsive Element (CRE) in Parathyroid Hormone and cAMP Induction of Human Interleukin-6 Promoter Activity. J Hard Tissue Biol 19: 65-74, 2010

10. Smith PK,Krohn RI, Hermanson GT, Mailia AK, Gartner FH, Provenzano MD, Fujimoto EK, Goeke NM, Oslson BJ and Klenk, DC. Measurement of protein using bicinchoninic acid. Analytic Biochem 150: 76-85, 1985

11. Sharpiro DJ, Sharp PA, Wahli, WW and Keller MJ. A highefficiency HeLa cell nuclear transcription extract. DNA 7: 47-55, 1988

12. Jansen E, Ayoubi TAY, Meulemans SMP and van der Ven WJM. Cell type-specific protein-DNA interactions at the cAMP response elements of the prohormone convertase I promoter. Evidence for transactivators distinct from CREB/ ATF family members. J Biol Chem 272: 2500-2508, 1997 